\title{
Angular Distribution and Polarization of Superradiant Emission from Atomic Ensembles
}

\author{
Verne Louis Jacobs \\ Center for Computational Materials Science, Code 6390, Materials Science and Technology Division, Naval Research Laboratory, \\ Washington, D. C., U.S.A \\ Email address: \\ Verne.Jacobs@nrl.navy.mil \\ To cite this article: \\ Verne Louis Jacobs. Angular Distribution and Polarization of Superradiant Emission from Atomic Ensembles. World Journal of Applied \\ Physics. Vol. 5, No. 1, 2020, pp. 1-14. doi: 10.11648/j.wjap.20200501.11
}

Received: March 1, 2020; Accepted: March 13, 2020; Published: April 1, 2020

\begin{abstract}
A density-matrix approach is developed to provide a theoretical description of the intensity, angular distribution, and polarization of superradiative emission from an ensemble of many-electron atomic systems. The many-electron atomic systems are described as cooperatively interacting by means of forces that can be long range. Particular emphasis is given to the coherent excitation of the collective atomic-ensemble states, which may be produced by incident laser radiation. The initial excitation and spontaneous emission processes may be described as independent. Both frequency-domain and time-domain formulations of the density-matrix approach are developed. The collective atomic-ensemble states are specified in a detailed hyperfine representation, corresponding to successively coupling the individual hyperfine angular momenta $\mathrm{F}$ pertaining to the many-electron atoms. A less detailed fine-structure angular-momentum representation may also be used. In the densityoperator approach, account can be taken of the coherent excitation of a particular subspace of the initial atomic-ensemble states. For a comprehensive and unified development of time-domain (equation-of-motion) and frequency-domain (resolventoperator) formulations, a reduced-density-matrix (quantum-open-systems) approach is introduced. The non-equilibrium atomic-ensemble-state kinetics and the homogeneous spectral-line shapes can thereby be systematically and self-consistently determined. The collective atomic-ensemble states may be obtained using a variety of different methods. These states can be determined using a dressed-state approach, in which the required states are calculated in the presence of an electromagnetic field.
\end{abstract}

Keywords: Superradiance, Atomic Ensembles, Density Matrix, Coherence

\section{Introduction}

Superradiant emission was first described by Dicke [1]. Due to the cooperative nature of the interaction among the individual members of an ensemble of $\mathrm{N}$ quantum systems, e. g., many-electron atoms, the intensity of superradiant emission can be proportional to $\mathrm{N}^{2}$. Moreover, the cooperative interaction can produce a large, superradiant dipole. A related phenomenon, known as subradiance, has also been investigated [2]. In the present investigation, we consider the angular distribution and polarization of superradiant emission. The angular distribution and polarization measurements can provide more detailed information, which would be unobtainable in a spectroscopy measurement of only the total intensity. The theoretical analysis of the total intensities, angular distributions, and polarizations can be developed using a density-matrix approach [3]. For a comprehensive theoretical description, it is necessary to employ a reduced-density-matrix (quantumopen-systems) approach, by means of which the influence of environmental decoherence and relaxation processes, together with the corresponding spectral-line broadening mechanisms, can be systematically and self-consistently incorporated [4].

A. Density-Matrix Approach

By means of a density-matrix approach, a general quantum-mechanical description of the intensity, angular distribution, and polarization of superradiant emission from an ensemble of $\mathrm{N}$ many-electron atomic systems can be developed. Account can thereby be taken of the coherent excitation of the atomic-ensemble states, which can be produced by incident lasers. Accordingly, it is advantageous 
to adapt a previously developed density-matrix description for the investigation atomic radiative emission, where the primary emphasis was directed at the influence of external electric and magnetic fields [3]. In the density-matrix description of the intensity, angular distribution, and polarization of superradiant emission, account can be taken of a general set of steady-state or time-varying (possibly coherent) atomic-ensemble excitation and de-excitation processes.

In the theoretical description of the angular distribution and polarization of atomic radiative emission, it is desirable to distinguish between a restricted polarization-densitymatrix description [3], which is rigorously applicable only to an isolated atomic system combined (or entangled) with the relevant (observable) modes of the quantized electromagnetic field, and a more comprehensive quantum-open-systems reduced-density-matrix formulation [4], in which the influence of a larger system (environment) of charged particles and photons can be systematically and selfconsistently incorporated. In the reduced-density-matrix formulation, the environmental collisional and radiative interactions are treated in terms of decoherence and relaxation processes, together with the associated spectralline broadening mechanisms.

B. Earlier Investigations for Directed-Electron Excitation

The ordinary Hilbert-space quantum theory of the intensity, angular distribution, and polarization of radiative emission, following directed-electron collisional excitation of an isolated atomic system, was first presented by Oppenheimer [5] and subsequently refined by Percival and Seaton [6], who also included an extensive set of references to earlier related investigations. A polarization-density-matrix formulation of the theory of radiative emission has been presented by Inal and Dubau for ordinary bound-bound atomic transitions [7] and subsequently extended for application to the dielectronic-recombination radiation process [8]. A polarization-density-matrix description for dielectronic recombination radiation has been developed by Shlyaptseva, Urnov, and Vinogradov [9, 10] and applied by Shlyaptseva et al. [11]. Spectroscopic observations of radiative emission from atomic transitions excited by electrons spirally in magnetic fields has been treated by Gu, Savin, and Beiersdorfer [12], using a polarization-densitymatrix approach. In a previously developed polarizationdensity-matrix description, which was primarily directed at bound-bound atomic radiative transitions [3], it has been found to be advantageous to exploit the angular-momentum methods and techniques advanced in earlier density-matrix descriptions of the angular distribution and polarization phenomena that can occur in single-photon and multi-photon atomic ionization processes [13, 14], as well as those adopted in the density-matrix description of the angular distribution and polarization of atomic radiative emission developed by Inal and Dubau [7, 8].

\section{Reduced-Density-Matrix Approach}

The more comprehensive reduced-density-matrix formulation can be developed using a quantum-open-systems approach [4]. A compact formulation is achieved by adopting the Liouville-space operator representation. This formulation can provide a detailed non-equilibrium quantum-statistical description of the angular distribution and polarization of radiative emission from an atomic ensemble for a general set of steady-state or time-varying (possibly coherent) excitation and de-excitation processes involving the atomic-ensemble states, under the influence of environmental collisional and radiative decoherence and relaxation processes.

D. Many-Electron Atomic-Ensemble States

In order to provide precise interpretations and theoretical predictions for spectroscopic observations of superradiant emission, it will be necessary to carry out reliable calculations for the relevant collective quantum states of the ensemble of many-electron atomic systems. Separate calculations should be performed for different assumptions regarding the possible influence of the incident laser fields in the excitation process. Accordingly, it might be appropriate to introduce a representation corresponding to "dressed" atomic-ensemble states, which would depend on the characteristics of the incident electromagnetic field.

E. Organization

The remainder of this paper is organized as follows. In Section II, we present the steady-state polarization densitymatrix description of superradiant emission, emphasizing that a realistic treatment of the superradiant emission process is expected to require the incorporation of initial-state coherences. In Section III, a time-dependent description of superradiant emission is presented. In Section IV, we discuss the representation of the atomic-ensemble states. In Section $\mathrm{V}$, we present a more comprehensive reduced-densityoperator approach, considering the influence of environmental decoherence and relaxation processes on both the atomic-ensemble states and the spectral-lines shapes. Our conclusions and future planes are presented in Section VI.

\section{Steady-State Polarization Density-Matrix Description}

The steady-state (time-independent) description of the radiative emission from an ensemble of many-electron atomic systems following photon (laser) excitation can be investigated on the bases of the following expression for the matrix elements of the photon density operator $\rho^{\mathrm{R}}$ :

$$
<\overrightarrow{\mathrm{k}} \lambda\left|\rho^{\mathrm{R}}\right| \overrightarrow{\mathrm{k}}^{\prime} \lambda^{\prime}>=\sum_{\mathrm{f}, \mathrm{i}, \mathrm{i}^{\prime}}<\gamma_{\mathrm{f}}, \overrightarrow{\mathrm{k}} \lambda|\mathrm{T}| \gamma_{\mathrm{i}}, 0><\gamma_{\mathrm{i}}\left|\rho^{\mathrm{A}}\right| \gamma_{\mathrm{i}^{\prime}}><\gamma_{\mathrm{i}^{\prime}}, 0|\mathrm{~T}| \gamma_{\mathrm{f}}, \overrightarrow{\mathrm{k}} \lambda^{\prime}>
$$

The angular frequency $\omega$ of the emitted electromagnetic radiation is given in terms of the magnitude $\mathrm{k}$ of the photon wave-vector $\overrightarrow{\mathrm{k}}$ by means of the free-space relation $\omega=\mathrm{kc}$. The angular distribution will be obtained from the matrix elements that are diagonal with respect to the photon wavevector. The photon-helicity quantum numbers, which represent the projections of the intrinsic spin of the spin-1 
quanta along the propagation direction, may have only the numerical values $\lambda, \lambda^{\prime}= \pm 1$, corresponding the right $(\lambda=-1)$ and left $(\lambda=+1)$ circular polarization. An alternative linearpolarization representation can be employed if desired. The summations over $f$, $i$, and i' will be understood to include only the quantum numbers specifying degenerate or nearly degenerate sub-states of the final and initial collective atomic-ensemble states in the radiative transitions $\gamma_{\mathrm{i}} \rightarrow \gamma_{\mathrm{f}}$. The degenerate sub-states of the individual atoms can be specified in terms of the usual angular-momentum projection (or magnetic) quantum numbers, either in the fine-structure $\left(\mathrm{JM}_{\mathrm{J}}\right)$ representation or in the more detailed hyperfine $\left(\mathrm{FM}_{\mathrm{F}}\right)$ representation. In this investigation, we shall adopt the more detailed hyperfine representation. The initial excitation process and the subsequent radiative emission process will be assumed to be independent events, and only single-photon emission processes will be taken into consideration.

The initial-state atomic density matrix $\rho^{A}$ could be determined on the basis of a steady-state Master equation. More generally, a time-dependent Master equation can be employed as a starting point. A detailed discussion of the time-dependent description will be presented below and also in our more comprehensive reduced-density-matrix formulation, which will facilitate the systematic incorporation of the influence of the multitude of environmental interactions. In the lowest-order perturbationtheory (Born) and short-memory-time (Markov) approximations, which are conventionally made in the treatment of environmental interactions, the equation-ofmotion for the diagonal matrix elements of $\rho^{A}$ (corresponding to the collective atomic-ensemble state populations) can be expressed in terms of the familiar (timeindependent) rates for all possible transitions between pairs of collective atomic-ensemble states. Including the nondiagonal matrix elements (corresponding to the coherences) in the equation-of-motion description, one encounters interference terms involving the individual transition amplitudes.

The steady-state spectral intensity, angular distribution, and polarization of the electromagnetic radiation that is emitted in the transitions $\gamma_{\mathrm{i}} \rightarrow \gamma_{\mathrm{f}}$ of an ensemble of manyelectron atoms can be systematically determined from a knowledge of the photon-polarization density operator $\rho^{\mathrm{R}}$. In terms of the transition operator $\mathrm{T}$, whose lowest-order contribution is given simply by the electromagneticinteraction operator $\mathrm{V}$, the quantum-electrodynamics theory of the single-photon emission process, in the density-operator representation of the atomic-ensemble states, can be employed to express the matrix elements of the photonpolarization density operator $\rho^{R}$ in a general form [15-23]:

The transition operator $\mathrm{T}$ can be evaluated using the Lippmann-Schwinger relationship, which can be expressed as $\mathrm{T}=\mathrm{V}+\mathrm{VGV}=\mathrm{V}+\mathrm{VG}_{0} T$. The full and zero-order resolvent (or Green) operators are defined by $\mathrm{G}=$ $\left[\mathrm{E}-\mathrm{H}_{0}-\mathrm{V}+\mathrm{i} \epsilon\right]^{-1}$ and $\mathrm{G}_{0}=\left[\mathrm{E}-\mathrm{H}_{0}+\mathrm{i} \epsilon\right]^{-1}$, where $\mathrm{H}_{0}$ is the Hamiltonian operator describing the unperturbed (isolated) atomic ensemble combined with that for the free (vacuum) electromagnetic field and $\mathrm{V}$ is the electromagnetic interaction. The electromagnetic interaction can be taken as a first approximation to be the dipole interaction. However, higher multipole-components can be systematically included. The appropriate asymptotic boundary condition can be imposed by taking the $\epsilon \rightarrow 0$ limit. The description of singlephoton emission processes based on Eq. (1), retaining only the lowest-order contribution in the perturbation expansion for the transition operator $\mathrm{T}$, is expected to be adequate for narrow, isolated emission lines or for blended emission features, whose individual spectral-line profiles may not be resolvable. In order to determine the precise spectral distribution of the possibly overlapping components, it will be necessary to retain a set of high-order contributions in the perturbation contributions. These high-order contributions give rise to the frequency shifts and the spectral widths of the radiative emission lines. The more comprehensive reduceddensity-matrix formulation will provide a systematic approach for the investigation of the spectral-line shape problem, taking into account the environmental interactions.

A. Individual Atomic-Ensemble Eigenstate Representation

Since polarization is intimately related to angular momentum, it is advantageous as a starting point to adopt an angular-momentum representation for the eigenstates of the many-electron atomic ensemble. An electromagneticmultipole expansion for the quantized radiation field will also be introduced. It then is apparent that the collective initial states pertaining to the many-electron atoms comprising the ensemble can be expanded in the detailed hyperfine-structure representation as follows:

$$
\left|\gamma \mathrm{i}>=\sum_{\Delta \mathrm{i} \mathrm{Fi} \mathrm{M}_{\mathrm{F}_{\mathrm{i}}}}\right| \Delta \mathrm{i} \mathrm{Fi} \mathrm{M}_{\mathrm{F}_{\mathrm{i}}}><\Delta \mathrm{iFi} \mathrm{M}_{\mathrm{F}_{\mathrm{i}}} \mid \gamma \mathrm{i}>
$$

Here $\mathrm{Fi}$ is the total angular momentum, $\mathrm{M}_{\mathrm{F}_{\mathrm{i}}}$ is the projection (or component) of this angular momentum along a suitably chosen quantization axis, and $\Delta \mathrm{i}$ denotes the set of remaining quantum numbers. Accordingly, the hyperfinestructure representation is characterized by the total (combined electronic and nuclear) angular momentum $\mathrm{F}=\mathrm{J}+\mathrm{I}$, where $\mathrm{J}$ represents the total electronic angular momentum and $\mathrm{I}$ is the total nuclear contribution. These angular-momentum quantum numbers are obtained by successively coupling the angular-momentum quantum numbers pertaining to the individual many-electron atomic systems comprising the ensemble. The individual final eigenstates of the atomic ensemble can be represented by an expansion in the same form as Eq. (2). We emphasize that the complete basis set of individual many-electron atomic states should ideally include the entire sets of the discrete bound states, the autoionizing resonances, and the non-resonance electron-ion-scattering (continuum-channel) states. This complete basis set can then be employed to represent the initial and final collective eigenstates of the many-electron atomic ensemble in the cooperative radiative emission process. Moreover, these collective eigenstates may be expanded in a basis consisting of (possibly symmetrized or anti-symmetrized) products of the individual many-electron atomic basis states. A detailed discussion of these collective states will be presented below.

In simplified atomic-structure calculations, only a relatively small set of low-lying bound eigenstates is 
normally considered. For an accurate determination of the spectral patterns, highly excited bound and continuum individual atomic eigenstates must be taken into account.

The atomic-state representation based on eigenstates of either the total electronic angular momentum $\mathrm{J}$ or the total angular momentum $\mathrm{F}$ has sufficient generality to accommodate a relativistic many-electron Hamiltonian description, and the corresponding individual atomic wave functions can be determined using existing relativistic multiconfiguration atomic-structure computer programs. However, it is well known that the relativistic many-electron atomicstructure problem cannot be described in terms of a closedform configuration-space Hamiltonian operator. The existing relativistic many-electron atomic-structure computer programs are based on the retention of only the lowest-order (Feynman-diagram) contributions in the perturbation expansion of quantum electrodynamics.

$$
<\Delta_{\mathrm{f}} \mathrm{F}_{\mathrm{f}} \mathrm{M}_{\mathrm{F}_{\mathrm{f}}}, \overrightarrow{\mathrm{k}} \lambda|\mathrm{T}| \Delta_{\mathrm{i}} \mathrm{F}_{\mathrm{i}} \mathrm{M}_{\mathrm{F}_{\mathrm{i}}}, 0>=\sum_{\mathrm{j}} \sum_{\mathrm{m}}\left(\frac{2 \mathrm{j}+1}{4 \pi}\right)^{1 / 2} \mathrm{~A}(\mathrm{j}) \mathrm{D}_{\lambda, \mathrm{m}}^{(\mathrm{j})}(\widehat{\mathrm{k}}) \times(-1)^{\mathrm{m}}<\Delta_{\mathrm{f}} \mathrm{F}_{\mathrm{f}} \mathrm{M}_{\mathrm{F}_{\mathrm{f}}}\left|\widetilde{\mathrm{Q}}_{-\mathrm{m}}^{(\mathrm{j})}\right| \Delta_{\mathrm{i}} \mathrm{F}_{\mathrm{i}} \mathrm{M}_{\mathrm{F}_{\mathrm{i}}}>
$$

Here $\widetilde{\mathrm{Q}}_{-\mathrm{m}}^{(\mathrm{j})}$ denotes the irreducible spherical-tensor form of the effective electromagnetic-multipole-moment operator for the ensemble of many-electron atomic systems. The lowestorder perturbation-theory component, which corresponds to the retention of only the electromagnetic-interaction operator $\mathrm{V}$ in the expression for the transition operator $\mathrm{T}$, is the usual electromagnetic-multipole-moment tensor operator $Q_{-m}^{(j)}$. The higher-order components may be viewed as corresponding to the radiative corrections that are predicted by quantumelectrodynamical perturbation theory, and these higher-order components represent the contributions from the various nonlinear electromagnetic processes arising from the absorption and emission of virtual photons and the creation and destruction of intermediate many-electron atomic states. It should be pointed out that the irreducible spherical tensor representation employed in Eq. (3) is valid for both the relativistic and the non-relativistic forms of the electromagnetic interaction. The quantities $D_{\lambda, m}^{(j)}(\widehat{k})$ designate
B. Multipole Expansion of the Electromagnetic Interaction The photon angular-distribution and polarization parameters are most naturally defined with respect to the direction of spectroscopic observation. In the theoretical analysis, however, it is more convenient to relate these parameters to the fundamental electromagnetic-transitions amplitudes, which are most naturally defined with respect to a suitably selected atomic quantization axis. The desired transformation is automatically introduced as a result of the irreducible spherical-tensor expansion of the electromagnetic interaction, as described in detail by Fano and Racah [18] and also by Berestetskii, Lifshitz, and Pitaevskii [19]. By means of this transformation, an irreducible spherical-tensor expansion is obtained for the electromagnetic-transition matrix elements, in terms of the matrix elements of effective electric and magnetic multipole operators. This expansion can be expressed in the form $[18,19]$ :

the matrix elements $D_{\lambda, m}^{(j)}(\phi, \vartheta, 0)$ of the Wigner rotation operator corresponding to the desired coordinate rotation. The multiplying factors $A(j)$ are defined individually for the various electromagnetic-multipole components. Explicit expressions for these multiplying factors have been presented by Berestetskii, Lifshitz, and Pitaevskii [19]. An expansion in the same form as Eq. (3) has also been employed by Inal and Dubau [7] for polarized radiative emission, taking into account all electromagnetic multipole contributions in lowest-order perturbation theory. The photon eigenstate representation characterized by linear momentum and intrinsic spin is thereby replaced by the alternative representation based on angular momentum and parity.

The matrix elements of the effective electromagneticmultipole-moment operator can be evaluated in the angularmomentum representation, in terms of Wigner 3-j symbols and reduced matrix elements of the effective electromagneticmultipole moment, by means of the Wigner-Eckart theorem:

$$
<\Delta_{\mathrm{f}} \mathrm{F}_{\mathrm{f}} \mathrm{M}_{\mathrm{F}_{\mathrm{f}}}\left|\widetilde{\mathrm{Q}}_{\mathrm{m}}^{(\mathrm{j})}\right| \Delta_{\mathrm{i}} \mathrm{F}_{\mathrm{i}} \mathrm{M}_{\mathrm{F}_{\mathrm{i}}}>=(-1)^{\mathrm{F}_{\mathrm{f}}-\mathrm{M}_{\mathrm{F}_{\mathrm{f}}}}\left(\begin{array}{ccc}
\mathrm{F}_{\mathrm{f}} & \mathrm{j} & \mathrm{F}_{\mathrm{i}} \\
-\mathrm{M}_{\mathrm{F}_{\mathrm{f}}} & \mathrm{m} & \mathrm{M}_{\mathrm{F}_{\mathrm{i}}}
\end{array}\right)\left(\Delta_{\mathrm{f}} \mathrm{F}_{\mathrm{f}}\left\|\widetilde{\mathrm{Q}}^{(\mathrm{j})}\right\| \Delta_{\mathrm{i}} \mathrm{F}_{\mathrm{i}}\right)
$$

In order to take into account both the electric and the magnetic multipole contributions associated with a given value of $\mathrm{j}$, the effective electromagnetic-multipole-moment operator $\widetilde{\mathrm{Q}}_{\mathrm{m}}^{(\mathrm{j})}$ should be defined to include the components associated with all permissible values of the photon parity. In contrast with the photon helicity $\lambda$, which can have only the values \pm 1 , the angular-momentum projection $\mathrm{m}$ is not limited to the values \pm 1 . The symmetry (or angular-momentum conservation) information is completely represented by the 3$\mathrm{j}$ symbols, while the dynamical information is entirely contained in the reduced matrix elements of the effective electromagnetic-multipole-moment operators.

C. Photon-Polarization Density Matrix Allowing for Coherent Excitation Processes

For ordinary bound-bound atomic radiative transitions, an expression for the photon-polarization density matrix has been obtained as Eq. [3]. In the derivation of this expression, only the lowest-order contribution, corresponding to the electromagnetic-interaction operator $\mathrm{V}$, was retained in the perturbation-theory expansion of the electromagnetictransition operator $\mathrm{T}$. In order to provide a more detailed spectral description for radiative transitions, as well as to incorporate quantum-mechanical interference phenomena, it will be necessary to consider the entire electromagnetictransition operator T. Accordingly, we now introduce the effective electromagnetic-multipole-moment operators $\widetilde{\mathrm{Q}}_{\mathrm{m}}^{(\mathrm{j})}$ based on Eq. (3). Considering only the photon-polarization density matrix elements that are diagonal in the photon wavevector, we obtain the following expression: 


$$
\begin{aligned}
& <\lambda\left|\rho^{\mathrm{R}}\right| \lambda^{\prime} \geq \sum_{\mathrm{i}, \mathrm{i}^{\prime}, \mathrm{f}}<\gamma_{\mathrm{i}}\left|\rho^{\mathrm{A}}\right| \gamma_{\mathrm{i}^{\prime}}>
\end{aligned}
$$

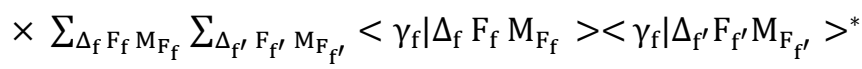

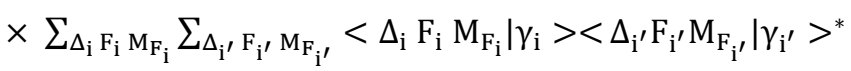

$$
\begin{aligned}
& \times \sum_{\mathrm{j}, \mathrm{m}} \sum_{\mathrm{j}^{\prime}, \mathrm{m}^{\prime}} \sum_{\mathrm{J}, \mathrm{M}, \mathrm{M}^{\prime}}\left(\Delta_{\mathrm{f}} \mathrm{F}_{\mathrm{f}}\left\|\widetilde{\mathrm{Q}}^{(\mathrm{j})}\right\| \Delta_{\mathrm{i}} \mathrm{F}_{\mathrm{i}}\right)\left(\Delta_{\mathrm{f}^{\prime}} \mathrm{F}_{\mathrm{f}^{\prime}}\left\|\widetilde{\mathrm{Q}}^{\left(\mathrm{j}^{\prime}\right)}\right\| \Delta_{\mathrm{i}^{\prime}} \mathrm{F}_{\mathrm{i}^{\prime}}\right)^{*} \\
& \times\left(\frac{1}{4 \pi}\right)(2 \mathrm{j}+1)^{1 / 2}\left(2 \mathrm{j}^{\prime}+1\right)^{1 / 2}(2 \mathrm{~J}+1)^{1 / 2} \mathrm{~A}(\mathrm{j}) \mathrm{A}\left(\mathrm{j}^{\prime}\right)^{*}
\end{aligned}
$$

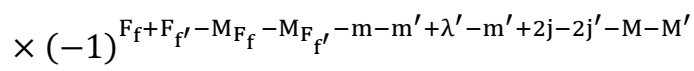

$$
\begin{aligned}
& \times\left(\begin{array}{ccc}
\mathrm{F}_{\mathrm{f}} & \mathrm{j} & \mathrm{F}_{\mathrm{i}} \\
-\mathrm{M}_{\mathrm{F}_{\mathrm{f}}} & -\mathrm{m} & \mathrm{M}_{\mathrm{F}_{\mathrm{i}}}
\end{array}\right)\left(\begin{array}{ccc}
\mathrm{F}_{\mathrm{f}^{\prime}} & \mathrm{j}^{\prime} & \mathrm{F}_{\mathrm{i}^{\prime}} \\
-\mathrm{M}_{\mathrm{F}_{\mathrm{f}^{\prime}}} & -\mathrm{m}^{\prime} & \mathrm{M}_{\mathrm{F}_{\mathrm{i}^{\prime}}}
\end{array}\right) \\
& \times\left(\begin{array}{ccc}
j & j^{\prime} & \mathrm{J} \\
\lambda & -\lambda^{\prime} & -\mathrm{M}
\end{array}\right)\left(\begin{array}{ccc}
\mathrm{j} & \mathrm{j}^{\prime} & \mathrm{J} \\
\mathrm{m} & -\mathrm{m}^{\prime} & -\mathrm{M}
\end{array}\right) \mathrm{D}_{\mathrm{M}, \mathrm{M}^{\prime}}^{(\mathrm{J})}(\widehat{\mathrm{k}}) \text {. }
\end{aligned}
$$

The summations over $\mathrm{f}$, $\mathrm{i}$, and $\mathrm{i}^{\prime}$ are to be taken over quantum numbers specifying degenerate or nearly degenerate substates of the final and initial collective states of the ensemble of many-electron atoms. In the derivation of this expression, the product of two Wigner rotation matrices, with ranks $\mathrm{j}$ and $\mathrm{j}^{\prime}$, has been expanded as a summation of Wigner rotation matrices corresponding to the total angular momentum $\mathrm{J}$, each of which is multiplied by two additional 3-j symbols. Although the primary interest has been in electric-dipole transitions, it is advantageous to retain at the beginning of the description the general form that is applicable for arbitrary electromagnetic-multipole interactions, including the interference between different multipole amplitudes. The general, non-perturbative expression for the effective electromagnetic-multipole-moment operators $\widetilde{\mathrm{Q}}^{(\mathrm{j})}$ can be expressed in terms of the entire electromagnetic-transition operator T. In the lowest-order perturbation-theory approximation for the effective electromagnetic-multipolemoment operators $\widetilde{Q}^{(j)}$, our result is found to be in agreement with that obtained by Inal and Dubau [7].

It should also be emphasized that the general expression

$$
\begin{gathered}
<\lambda\left|\rho^{\mathrm{R}}\right| \lambda^{\prime}>=\sum_{\mathrm{M}_{\mathrm{F}_{i}}} \mathrm{~N}\left(\Delta_{\mathrm{i}} \mathrm{F}_{\mathrm{i}} \mathrm{M}_{\mathrm{F}_{\mathrm{i}}}\right) \sum_{j, j^{\prime}, J}(-1)^{\mathrm{F}_{\mathrm{i}}+\mathrm{j}+\mathrm{j}^{\prime}+\lambda^{\prime}-\mathrm{M}_{\mathrm{F}_{\mathrm{i}}}} \\
\times\left[(2 \mathrm{j}+1)\left(2 \mathrm{j}^{\prime}+1\right)(2 \mathrm{~J}+1) / 4 \pi\right]^{1 / 2} \mathrm{~A}(\mathrm{j}) \mathrm{A}\left(\mathrm{j}^{\prime}\right)^{*} \\
\times\left(\Delta_{\mathrm{f}} \mathrm{F}_{\mathrm{f}}\left\|\widetilde{Q}^{(j)}\right\| \Delta_{\mathrm{i}} \mathrm{F}_{\mathrm{i}}\right)\left(\Delta_{\mathrm{f}} \mathrm{F}_{\mathrm{f}}\left\|\widetilde{Q}^{\left(j^{\prime}\right)}\right\| \Delta_{\mathrm{i}} \mathrm{F}_{\mathrm{i}}\right)^{*} \\
\times\left(\begin{array}{ccc}
J & j & \mathrm{j}^{\prime} \\
\lambda^{\prime}-\lambda & \lambda & -\lambda^{\prime}
\end{array}\right)\left(\begin{array}{ccc}
\mathrm{J} & \mathrm{F}_{\mathrm{i}} & \mathrm{F}_{\mathrm{i}} \\
0 & -\mathrm{M}_{\mathrm{F}_{\mathrm{i}}} & \mathrm{M}_{\mathrm{F}_{\mathrm{i}}}
\end{array}\right)\left\{\begin{array}{ccc}
J & \mathrm{~F}_{\mathrm{i}} & \mathrm{F}_{\mathrm{i}} \\
\mathrm{J}_{\mathrm{f}} & \mathrm{j}^{\prime} & \mathrm{j}
\end{array}\right\} \mathrm{Y}_{\lambda^{\prime}-\lambda}^{(\mathrm{J})(\hat{\mathrm{k}}) .}
\end{gathered}
$$

The diagonal matrix elements of $\rho^{\mathrm{A}}$, which correspond to the usual level population densities, are now denoted by $\mathrm{N}\left(\Delta_{\mathrm{i}} \mathrm{F}_{\mathrm{i}} \mathrm{M}_{\mathrm{F}_{\mathrm{i}}}\right)$. The condition $\mathrm{M}^{\prime}=0$ follows from the neglect of the non-diagonal matrix elements of $\rho^{\mathrm{A}}$. The Wigner rotation matrix elements $D_{M, M^{\prime}}^{(J)}(\hat{k})$ can then be reduced to the spherical harmonic functions $\mathrm{Y}_{\lambda^{\prime}-\lambda}^{(\mathrm{J})}(\hat{\mathrm{k}})$, for which the total angular momentum $J$ can assume only integer values. The dominant radiative emission process is usually assumed to involve only a single multipole component of the electromagnetic field, in which case $\mathrm{j}=\mathrm{j}^{\prime}$. Eqs. (5) and (6) are valid for interfering electromagnetic-multipole components, corresponding to $\mathrm{j} \neq \mathrm{j}^{\prime}$. This interference can have a more important effect on the angular distribution and polarization than on the total frequency-integrated photon intensity.

D. Irreducible Spherical-Tensor Representation of the Density Operators

By means of Eq. (5), the matrix elements of photonpolarization density operator have been expressed in a non- 
perturbative form, in terms of the reduced matrix elements of the effective electromagnetic-multipole-moment operators denoted by $\widetilde{Q}^{(j)}$. The matrix elements of the photonpolarization density operator $\rho^{\mathrm{R}}$ are expressed as functions of the photon-emission direction, in terms of which the photonhelicity quantum number $\lambda$ is defined. Following Fano and Racah [18], Happer [20, 21], Omont [22], and Baylis [23], this photon-polarization density operator $\rho^{\mathrm{R}}$ may be represented, as an expansion in terms of the irreducible spherical-tensor operators $\mathrm{T}_{\mathrm{M}}^{(\mathrm{J})}\left(\mathrm{j}, \mathrm{j}^{\prime}\right)$, as follows:

$$
\rho^{\mathrm{R}}=\sum_{\mathrm{j}, \mathrm{j}^{\prime}} \sum_{\mathrm{J}, \mathrm{M}, \mathrm{M}^{\prime}} \rho^{\mathrm{R}}\left(\mathrm{j}, \mathrm{j}^{\prime} ; \mathrm{J}, \mathrm{M}^{\prime}\right) \mathrm{T}_{\mathrm{M}}^{(\mathrm{J})}\left(\mathrm{j}, \mathrm{j}^{\prime}\right) \mathrm{D}_{\mathrm{M}, \mathrm{M}^{\prime}}^{(\mathrm{J})}(\hat{\mathrm{k}}) .
$$

In the Liouville-space Dirac notation, which will be adopted in the more comprehensive reduced-density-operator formulation outlined below, this expansion corresponds to a transformation from the representation of the uncoupled states $\mid j m, j^{\prime} m^{\prime} \gg$ to the alternative representation of the coupled states $\mid j, j^{\prime} ; J, M \gg$. The quantities $\rho^{R}\left(j, j^{\prime} ; J, M^{\prime}\right)$ are referred to as the irreducible spherical-tensor components of the photonpolarization density operator. These quantities are also known as state multipoles or statistical tensors. The photon-helicity quantum numbers are defined, with respect to the photon propagation direction, by means of the Wigner rotation matrices $\mathrm{D}_{\mathrm{M}, \mathrm{M}^{\prime}}^{(\mathrm{J})}(\hat{\mathrm{k}})$. The ordinary Hilbert-space matrix elements of the irreducible spherical-tensor operator $\mathrm{T}_{\mathrm{M}}^{(J)}\left(\mathrm{j}, \mathrm{j}^{\prime}\right)$ can be evaluated using the Wigner-Eckart theorem as follows:

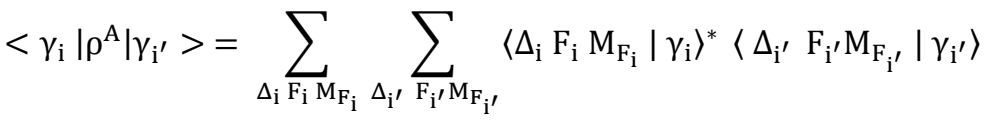

$$
\begin{aligned}
& \times \sum_{\mathrm{K}, \mathrm{N}} \rho^{\mathrm{A}}\left(\Delta_{\mathrm{i}} \Delta_{\mathrm{i}^{\prime}}, \mathrm{F}_{\mathrm{i}} \mathrm{F}_{\mathrm{i}^{\prime}} ; \mathrm{K}, \mathrm{N}\right)(-1)^{\mathrm{F}_{\mathrm{i}}-\mathrm{M}_{\mathrm{F}_{\mathrm{i}}}}(2 \mathrm{~K}+1)^{1 / 2}\left(\begin{array}{ccc}
\mathrm{F}_{\mathrm{i}} & \mathrm{K} & \mathrm{F}_{\mathrm{i}^{\prime}} \\
-\mathrm{M}_{\mathrm{F}_{\mathrm{i}}} & \mathrm{N} & \mathrm{M}_{\mathrm{F}_{\mathrm{i}}}
\end{array}\right) \text {. }
\end{aligned}
$$

By means of Eqs. (5), (7), (9), and (10), the irreducible density operator $\rho^{R}$ can be expressed in terms of the irreducible spherical-tensor components of the atomicensemble density operator $\rho^{A}$ and the reduced matrix elements describing the electromagnetic transition. A natural separation between the geometrical (or symmetry) and dynamical factors is thereby achieved.

E. Electric-Dipole Transitions

Although the importance of higher-order multipole (forbidden) radiative transitions from certain excited atomic states has been widely recognized, atomic systems have been customarily assumed to undergo spontaneous radiative decay spherical-tensor components of the photon-polarization

$$
<j \lambda\left|T_{M}^{(J)}\left(j, j^{\prime}\right)\right| j^{\prime} \lambda^{\prime}>=(-1)^{j-\lambda}(2 J+1)^{1 / 2}\left(\begin{array}{ccc}
j & J & j^{\prime} \\
-\lambda & M & \lambda^{\prime}
\end{array}\right) .
$$

The reduced matrix element of $\mathrm{T}_{\mathrm{M}}^{(\mathrm{J})}\left(\mathrm{j}, \mathrm{j}^{\prime}\right)$ is accordingly given by the factor $(2 \mathrm{~J}+1)^{1 / 2}$. The general expression for the irreducible spherical-tensor components $\rho^{\mathrm{R}}\left(\mathrm{j}, \mathrm{j}^{\prime} ; \mathrm{J}, \mathrm{M}^{\prime}\right)$ can be obtained simply by comparing Eqs. (5) and (7), employing Eq. (8), and the symmetry properties of the $3-\mathrm{j}$ symbols. This irreducible spherical-tensor representation is often advantageous for the photon density operator, because only a few multipole components of the electromagnetic field are usually required to provide an adequate description of the radiative-emission process.

In contrast to the photon density operator, the irreducible spherical-tensor representation of the atomic-ensemble state density operator, which may be expressed in the form:

$\rho^{\mathrm{A}}=\sum_{\Delta_{\mathrm{i}} \Delta_{\mathrm{i}^{\prime}}} \sum_{\mathrm{F}_{\mathrm{i}} \mathrm{F}_{\mathrm{i}^{\prime}}} \sum_{\mathrm{K}, \mathrm{N}} \rho^{\mathrm{A}}\left(\Delta_{\mathrm{i}} \Delta_{\mathrm{i}^{\prime}}, \mathrm{F}_{\mathrm{i}} \mathrm{F}_{\mathrm{i}^{\prime}} ; \mathrm{K}, \mathrm{N}\right) \mathrm{T}_{\mathrm{N}}^{(\mathrm{K})}\left(\mathrm{F}_{\mathrm{i}} \mathrm{F}_{\mathrm{i}^{\prime}}\right)$,

involves two separate expansions that ideally should include the complete basis set of unperturbed individual manyelectron atomic-ensemble eigenstates.

In order to express the irreducible spherical-tensor components of the photon-polarization density operator in terms of the atomic-ensemble irreducible spherical-tensor components $\rho^{\mathrm{A}}\left(\Delta_{\mathrm{i}} \Delta_{\mathrm{i}^{\prime}}, \mathrm{F}_{\mathrm{i}} \mathrm{F}_{\mathrm{i}^{\prime}} ; \mathrm{K}, \mathrm{N}\right)$, it is necessary employ the following transformation: predominantly by means of electric-dipole transitions. Moreover, the quantum-mechanical interference involving different electromagnetic-multipole components has usually been ignored.

1. Atomic-Ensemble State Coherences Ignored

As a first step, we will ignore initial atomic-ensemble state coherences, noting that this is not expected to be a valid assumption for cooperative radiative emission processes. The diagonal matrix elements of the photon-polarization density operator describing spontaneous electric-dipole emission, neglecting initial state coherences, can be expressed as follows:

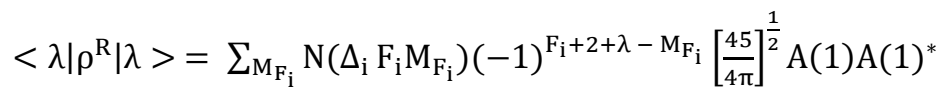

$$
\begin{aligned}
& \times\left(\Delta_{\mathrm{f}} \mathrm{F}_{\mathrm{f}}|| \mathrm{Q}^{(1)}|| \Delta_{\mathrm{i}} \mathrm{F}_{\mathrm{i}}\right)\left(\Delta_{\mathrm{f}} \mathrm{F}_{\mathrm{f}}|| \mathrm{Q}^{(1)}|| \Delta_{\mathrm{i}} \mathrm{F}_{\mathrm{i}}\right)^{*} \\
& \times\left(\begin{array}{ccc}
2 & 1 & 1 \\
0 & \lambda & -\lambda
\end{array}\right)\left(\begin{array}{ccc}
2 & \mathrm{~F}_{\mathrm{i}} & \mathrm{F}_{\mathrm{i}} \\
0 & -\mathrm{M}_{\mathrm{F}_{\mathrm{i}}} & \mathrm{M}_{\mathrm{F}_{\mathrm{i}}}
\end{array}\right)\left\{\begin{array}{ccc}
2 & \mathrm{~F}_{\mathrm{i}} & \mathrm{F}_{\mathrm{i}} \\
\mathrm{J}_{\mathrm{f}} & 1 & 1
\end{array}\right\} \mathrm{Y}_{0}^{2}(\hat{\mathrm{k}})
\end{aligned}
$$

This expression can be further evaluated as follows:

$$
<\lambda\left|\rho^{\mathrm{R}}\right| \lambda>=\sum_{\mathrm{M}_{\mathrm{i}}} \mathrm{N}\left(\Delta_{\mathrm{i}} \mathrm{F}_{\mathrm{i}} \mathrm{M}_{\mathrm{F}_{\mathrm{i}}}\right)(-1)^{\mathrm{F}_{\mathrm{i}}+2+\lambda-\mathrm{M}_{\mathrm{F}_{\mathrm{i}}}}
$$




$$
\begin{aligned}
& \times\left[\frac{45}{4 \pi}\right]^{\frac{1}{2}} \mathrm{~A}(1) \mathrm{A}(1)^{*}\left(\Delta_{\mathrm{f}} \mathrm{F}_{\mathrm{f}}|| \mathrm{Q}^{(1)}|| \Delta_{\mathrm{i}} \mathrm{F}_{\mathrm{i}}\right)\left(\Delta_{\mathrm{f}} \mathrm{F}_{\mathrm{f}}|| \mathrm{Q}^{(1)}|| \Delta_{\mathrm{i}} \mathrm{F}_{\mathrm{i}}\right)^{*} \\
& \times(-1)^{1-\lambda} \frac{3 \lambda^{2}-2}{\sqrt{1 \times 1 \times 3 \times 2 \times 5}}(-1)^{\mathrm{F}_{\mathrm{i}}-\mathrm{M}_{\mathrm{F}_{\mathrm{i}}}} \frac{3 \mathrm{M}_{\mathrm{F}_{\mathrm{i}}}{ }^{2}-\mathrm{F}_{\mathrm{i}}\left(\mathrm{F}_{\mathrm{i}}+1\right)}{\sqrt{\left(2 \mathrm{~F}_{\mathrm{i}}-1\right) \mathrm{F}_{\mathrm{i}}\left(2 \mathrm{~F}_{\mathrm{i}}+1\right)\left(\mathrm{F}_{\mathrm{i}}+1\right)\left(2 \mathrm{~F}_{\mathrm{i}}+3\right)}} \\
& \times\left\{\begin{array}{ccc}
2 & F_{i} & F_{i} \\
F_{f} & 1 & 1
\end{array}\right\} \sqrt{\frac{5}{16 \pi}}\left(3 \cos ^{2} \theta-1\right) .
\end{aligned}
$$

The conventional spontaneous radiative-emission rate (or Einstein A coefficient) can be obtained after carrying out the summation over the final magnetic substates $\mathrm{M}_{\mathrm{F}_{\mathrm{i}}}$ and the photon polarizations $\lambda$, together with the integration over the photon emission angles. The result thereby obtained can be expressed in the familiar form:

$$
\mathrm{A}_{\mathrm{r}}\left(\Delta_{\mathrm{i}} \mathrm{F}_{\mathrm{i}} \rightarrow \Delta_{\mathrm{f}} \mathrm{F}_{\mathrm{f}}\right)=\left(\frac{4}{3}\right)\left(\frac{\omega^{3}}{\hbar c^{3}}\right)\left(\frac{1}{2 \mathrm{~F}_{\mathrm{i}}+1}\right)\left|\left(\Delta_{\mathrm{f}} \mathrm{F}_{\mathrm{f}}|| \mathrm{Q}^{(1)}|| \Delta_{\mathrm{i}} \mathrm{F}_{\mathrm{i}}\right)\right|^{2} .
$$

Equations (12) and (13) have been obtained by introducing the electric-dipole approximation for the electromagnetic interaction and by assuming a uniform (statistical) distribution of the initial magnetic-substate populations.

2. Atomic-Ensemble State Coherences Included

It is expected that atomic-ensemble state coherences will play an important role in the cooperative radiative processes such as superradiant emission. If initial atomic-ensemble state coherences are now included, the diagonal matrix elements of the photon-polarization density operator describing spontaneous electric-dipole emission can be expressed as follows:

$$
\begin{aligned}
& \left.<\lambda \mid \rho^{\mathrm{R}}\right\rceil \lambda \geq \sum_{\mathrm{i}, \mathrm{i}^{\prime}, \mathrm{f}}<\gamma_{\mathrm{i}}\left|\rho^{\mathrm{A}}\right| \gamma_{\mathrm{i}^{\prime}}>
\end{aligned}
$$

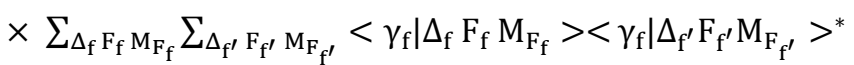

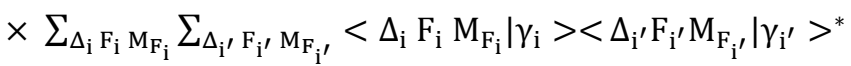

$$
\begin{aligned}
& \times \sum_{\mathrm{m}} \sum_{\mathrm{J}, \mathrm{M}, \mathrm{M}^{\prime}}\left(\Delta_{\mathrm{f}} \mathrm{F}_{\mathrm{f}}\left\|\widetilde{\mathrm{Q}}^{(1)}\right\| \Delta_{\mathrm{i}} \mathrm{F}_{\mathrm{i}}\right)\left(\Delta_{\mathrm{f}^{\prime}} \mathrm{F}_{\mathrm{f}^{\prime}}\left\|\widetilde{\mathrm{Q}}^{(1)}\right\| \Delta_{\mathrm{i}^{\prime}} \mathrm{F}_{\mathrm{i}^{\prime}}\right)^{*} \\
& \times\left(\frac{1}{4 \pi}\right)(3)^{1 / 2}(3)^{1 / 2}(2 \mathrm{~J}+1)^{1 / 2} \mathrm{~A}(1) \mathrm{A}(1)^{*}
\end{aligned}
$$

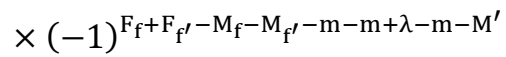

$$
\begin{aligned}
& \times\left(\begin{array}{ccc}
\mathrm{F}_{\mathrm{f}} & 1 & \mathrm{~F}_{\mathrm{i}} \\
-\mathrm{M}_{\mathrm{F}_{\mathrm{f}}} & -\mathrm{m} & \mathrm{M}_{\mathrm{F}_{\mathrm{i}}}
\end{array}\right)\left(\begin{array}{ccc}
\mathrm{F}_{\mathrm{f}^{\prime}} & 1 & \mathrm{~F}_{\mathrm{i}^{\prime}} \\
-\mathrm{M}_{\mathrm{F}_{\mathrm{f}^{\prime}}} & -\mathrm{m} & \mathrm{M}_{\mathrm{F}_{\mathrm{i}^{\prime}}}
\end{array}\right) \\
& \times\left(\begin{array}{ccc}
\mathrm{j} & \mathrm{j} & \mathrm{J} \\
\lambda & -\lambda & 0
\end{array}\right)\left(\begin{array}{ccc}
1 & 1 & \mathrm{~J} \\
\mathrm{~m} & -\mathrm{m} & 0
\end{array}\right) \mathrm{D}_{0, \mathrm{M}^{\prime}}^{(\mathrm{J})}(\hat{\mathrm{k}})
\end{aligned}
$$

This expression can be further evaluated as follows:

$$
\begin{aligned}
& <\lambda\left[\rho^{\mathrm{R}}\right\rceil \lambda \geq \sum_{\mathrm{i}, \mathrm{i}^{\prime}, \mathrm{f}}<\gamma_{\mathrm{i}}\left|\rho^{\mathrm{A}}\right| \gamma_{\mathrm{i}^{\prime}}>
\end{aligned}
$$

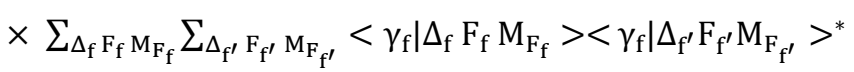

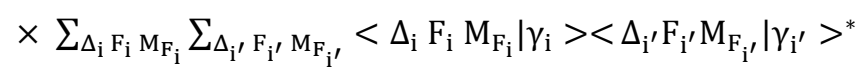

$$
\begin{aligned}
& \times\left(\Delta_{\mathrm{f}} \mathrm{F}_{\mathrm{f}}\left\|\widetilde{\mathrm{Q}}^{(1)}\right\| \Delta_{\mathrm{i}} \mathrm{F}_{\mathrm{i}}\right)\left(\Delta_{\mathrm{f}^{\prime}} \mathrm{F}_{\mathrm{f}^{\prime}}\left\|\widetilde{\mathrm{Q}}^{(1)}\right\| \Delta_{\mathrm{i}^{\prime}} \mathrm{F}_{\mathrm{i}^{\prime}}\right)^{*}\left(\frac{1}{4 \pi}\right)(3)^{1 / 2}(3)^{1 / 2} \mathrm{~A}(1) \mathrm{A}(1)^{*}
\end{aligned}
$$

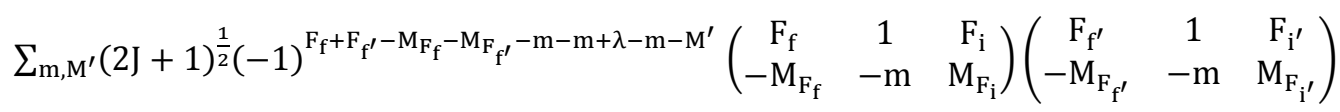

$$
\begin{aligned}
& {\left[\begin{array}{c}
\frac{1}{3}(-1)^{-\lambda-\mathrm{m}} \delta\left(\mathrm{M}^{\prime}, 0\right)+\frac{\lambda \mathrm{m}}{6}(-1)^{1-\lambda-\mathrm{m}}\left((-1)^{\mathrm{M}^{\prime}} \sqrt{\frac{4 \pi}{3}} \mathrm{Y}^{*}{ }_{1 \mathrm{M}^{\prime}(\theta \phi)}\right) \\
+ \\
\frac{1}{30}(-1)^{-\lambda-\mathrm{m}}\left(3 \lambda^{2}-2\right)\left(3 \mathrm{~m}^{2}-2\right)\left((-1)^{\mathrm{M}^{\prime}} \sqrt{\frac{4 \pi}{5}} \mathrm{Y}^{*}{ }_{2 \mathrm{M}^{\prime}(\theta \phi)}\right)
\end{array}\right]}
\end{aligned}
$$




\section{Time-Dependent Polarization Density- Matrix Description}

A time-dependent description of superradiative emission from an ensemble of many-electron atomic systems accompanying photon (laser) excitation can be developed on the basis of the following expression for the matrix elements of the time-dependent photon-polarization density operator $\rho^{\mathrm{R}}(\mathrm{t})$ :

$$
<\lambda\left|\rho^{\mathrm{R}}(\mathrm{t})\right| \lambda^{\prime}>=\sum_{\mathrm{f}, \mathrm{i}, \mathrm{i}^{\prime}}<\gamma_{\mathrm{f}}, \overrightarrow{\mathrm{k}} \lambda\left|\mathrm{U}\left(\mathrm{t}, \mathrm{t}_{0}\right)\right| \gamma_{\mathrm{i}}, 0><\gamma_{\mathrm{i}}\left|\rho^{\mathrm{A}}\left(\mathrm{t}_{0}\right)\right| \gamma_{\mathrm{i}^{\prime}}>\times<\gamma_{\mathrm{i}^{\prime}}, 0\left|\mathrm{U}\left(\mathrm{t}, \mathrm{t}_{0}\right)^{-1}\right| \gamma_{\mathrm{f}}, \overrightarrow{\mathrm{k}} \lambda^{\prime}>
$$

The initial-state many-atom-ensemble photon-polarization density operator by $\rho^{\mathrm{A}}\left(\mathrm{t}_{0}\right)$, which is specified at an appropriate intial time $t_{0}$, could be determined very generally by first solving a timedependent Master equation. A detailed discussion of the frequency-domain and time-domain formulations of this more comprehensive reduced-density-matrix description, which will enable the systematic incorporation of the influence of the multitude of environmental interactions, will be presented below. In the asymptotic limit, where $t_{0}=$ $-\infty$ and $t=+\infty$, the evolution operator $U\left(t, t_{0}\right)$ can be related to the transition operator $\mathrm{T}$ appearing in the timeindependent description based on Eq. (1).

The spectral intensity, angular distribution, and polarization of the electromagnetic radiation that is emitted in the transitions $\gamma_{i} \rightarrow \gamma_{f}$ of an ensemble of many-electron atoms can be systematically determined from a knowledge of the time-dependent photon-polarization density operator $\rho^{R}(t)$. In the Schrödinger picture, the time evolution operator $\mathrm{U}\left(\mathrm{t}, \mathrm{t}_{0}\right)$ can be related to the zero-order (unperturbed) Hamiltonian operator $\mathrm{H}_{0}$ and the electromagnetic-interaction operator $\mathrm{V}(\mathrm{t})$ as follows:

$$
\mathrm{i} \hbar \frac{\partial \mathrm{U}\left(\mathrm{t}, \mathrm{t}_{0}\right)}{\partial \mathrm{t}}=\left[\mathrm{H}_{0}+\mathrm{V}(\mathrm{t})\right] \mathrm{U}\left(\mathrm{t}, \mathrm{t}_{0}\right)
$$

The quantum-electrodynamics theory of the single-photon emission process, in the density-operator representation of the atomic-ensemble states, can be employed to express the matrix elements of the time-dependent photon-polarization density operator $\rho^{\mathrm{R}}(\mathrm{t})$ in a general form [15-23], utilizing Eq. (16).

The time evolution operator $U\left(t, t_{0}\right)$ can be evaluated using the expansion:

$$
\mathrm{U}\left(\mathrm{t}, \mathrm{t}_{0}\right)=\operatorname{Texp}\left[\left(\frac{-\mathrm{i}}{\hbar}\right) \int_{\mathrm{t}_{0}}^{\mathrm{t}} \mathrm{dt}^{\prime}\left(\mathrm{H}_{0}+\mathrm{V}\left(\mathrm{t}^{\prime}\right)\right)\right]=1+\sum_{\mathrm{n}=1}^{\infty}\left(\frac{1}{\mathrm{n} !}\right)\left(\frac{-\mathrm{i}}{\hbar}\right)^{\mathrm{n}} \prod_{\mathrm{m}=1}^{\mathrm{n}} \int_{\mathrm{t}_{0}}^{\mathrm{t}} \mathrm{dt}_{\mathrm{m}} \mathrm{T}\left(\mathrm{H}_{0}+\mathrm{V}\left(\mathrm{t}_{\mathrm{m}}\right)\right)
$$

where $\mathrm{T}$ denotes the time-ordering operator. We are particularly concerned with the many-atom states arising from the collective interaction of the atoms comprising the ensemble undergoing excitation in the presence of the incident laser fields. These states could be treated in a "dressed-state" representation induced by a classical timedependent field [24]. In the time-dependent description, it will not be necessary (and may not be appropriate) to assume that the excitation and radiative emission processes can be treated as independent events, a we did in the timeindependent description, since we can now include all modes of the time-dependent electromagnetic field, particularly the incident laser and vacuum fields, in the electromagnetic- interaction operator $\mathrm{V}(\mathrm{t})$.

It then is apparent that the collective initial and final states pertaining to the many-electron atoms comprising the ensemble, which appear in Eq. (16), can be expanded in the detailed hyperfine representation, as expressed by means of Eq. (2). We can now introduce an electromagnetic-multipole expansion of the time-evolution operator $U\left(t, t_{0}\right)$ given by Eq. (18). An irreducible spherical-tensor expansion is obtained for the matrix elements of the time-evolution operator $\mathrm{U}\left(\mathrm{t}, \mathrm{t}_{0}\right)$, in terms of the matrix elements of the timedependent effective electromagnetic-multipole-moment operators $\left.\overline{Q\left(t, t_{0}\right.}\right)_{-m}^{(j)}$, as follows:

$$
<\Delta_{\mathrm{f}} \mathrm{F}_{\mathrm{f}} \mathrm{M}_{\mathrm{F}_{\mathrm{f}}}, \overrightarrow{\mathrm{k}} \lambda\left|\mathrm{U}\left(\mathrm{t}, \mathrm{t}_{0}\right)\right| \Delta_{\mathrm{i}} \mathrm{F}_{\mathrm{i}} \mathrm{M}_{\mathrm{F}_{\mathrm{i}}}, 0>=\sum_{\mathrm{j}} \sum_{\mathrm{m}}\left(\frac{2 \mathrm{j}+1}{4 \pi}\right)^{1 / 2} \mathrm{~B}(\mathrm{j}) \mathrm{D}_{\lambda, \mathrm{m}}^{(\mathrm{j})}(\widehat{\mathrm{k}}) \times(-1)^{\mathrm{m}}<\Delta_{\mathrm{f}} \mathrm{F}_{\mathrm{f}} \mathrm{M}_{\mathrm{F}_{\mathrm{f}}} \mid \widetilde{\left.\mathrm{Q}_{\left(\mathrm{t}, \mathrm{t}_{0}\right.}\right)_{-\mathrm{m}}^{(\mathrm{j})} \mid} \Delta_{\mathrm{i}} \mathrm{F}_{\mathrm{i}} \mathrm{M}_{\mathrm{F}_{\mathrm{i}}}>.
$$

The multiplying factors $B(j)$, which are analogous to the factors $A(j)$ appearing in Eq. (3), may be determined by the requirement that the irreducible spherical-tensor forms of the time-dependent effective electromagnetic-multipole operators $\mathrm{Q}\left(\overline{\mathrm{t}, \mathrm{t}_{0}}\right)_{-\mathrm{m}}^{(\mathrm{j})}$ should reduce to the corresponding irreducible spherical-tensor forms of the time-independent effective electromagnetic-multipole-moment operators $\widetilde{\mathrm{Q}}_{-\mathrm{m}}^{\mathrm{j})}$ that have been introduced in Eq. (3) when the asymptotic limits giving rise to the transition operator are taken. The desired time dependence of the radiative emission will be introduced by means of the time-dependence of these effective electromagnetic-multipole-moment operators.

\section{Representation of Atomic-Ensemble States}

The quantum state that can be formed following singlephoton absorption by an ensemble of $\mathrm{N}$ many-electron atomic systems can be represented as follows:

$$
\left|\Phi_{\mathrm{N}}^{1}\right\rangle=\frac{1}{\sqrt{\mathrm{N}}} S \sum_{\mu=1}^{\mathrm{N}} \exp ^{\overrightarrow{\mathrm{lk} \cdot \mathrm{r}_{\mu}}}\left\langle\mathrm{e}_{\mu} \mathrm{g}^{\otimes(\mathrm{N}-1)} \mid \Phi_{\mathrm{N}}\right\rangle .
$$

In this representation, we have assumed that only one of the $\mathrm{N}$ atomic systems has been exited to the state $\mid \mathrm{e}>$ at the position $\overrightarrow{r_{\mu}}$ while the remaining atomic systems are in their ground state $\mid g>$. The symbol $\mathrm{S}$ denotes the symmetrization 
operation. In some cases, the corresponding antisymmetrization operation should be employed. In order to obtain the detailed hyperfine-structure basis states that have been denoted by $|\gamma\rangle$ in the angular distribution and polarization analysis that we have presented above, it is necessary to successively couple the individual hyperfinestructure angular-momentum quantum numbers pertaining to the separate $\mathrm{N}$ many-electron atomic systems to form the eigenstates of the total angular momentum F, which is the sum of the total electronic angular momentum $\mathrm{J}$ and nuclear angular momentum I. Although not explicitly indicated, this coupling operation will be understood to be carried out.
The cooperative interaction involving the individual atomic systems should be incorporated by means of a diagonalization procedure. In this diagonalization procedure, it is expected to be necessary to include more general basis states, corresponding to the excitation of two or more many-electron atomic systems. Accordingly, we should consider a larger manifold of basis states of the ensemble of $\mathrm{N}$ many-electron states to include those states that can be formed by the absorption of $\mathrm{M}>1$ photons and the excitation of more than one of the $\mathrm{N}$ atomic systems. The more general basis states can be expressed by means of the following representation [25]:

$$
\left|\Phi_{n}^{(M)}>=\frac{1}{\sqrt{\mathrm{C}_{\mathrm{M}}^{\mathrm{N}}}} \prod_{j=1}^{\mathrm{M}} \sum_{\mu_{\mathrm{j}}=\mu_{\mathrm{j}-1}+1}^{\mathrm{N}-\mathrm{M}+\mathrm{j}} \exp ^{\mathrm{i} \overrightarrow{\mathrm{k} \cdot \mathrm{R}_{\mathrm{M}}}} \exp ^{\mathrm{i} \frac{2 \mathrm{n} \pi\{\mathrm{f}(\overrightarrow{\mu)-1]}}{\mathrm{C}_{\mathrm{M}}^{\mathrm{N}}}}\right| \Psi_{\mathrm{N}}^{(\mathrm{M})}(\vec{\mu}>>
$$

A symmetric state can be formed, in which $M$ excited states and $\mathrm{N}-\mathrm{M}$ ground states are symmetrically distributed among all atoms with equal probabilities $\frac{1}{\mathrm{C}_{\mathrm{M}}^{\mathrm{N}}}$, where the symbol $C$ denotes the binominal coefficients. $n \in\left[1, C_{M}^{N}\right]$ and $\mathrm{f}\left(\overrightarrow{\mu)}=\sum_{\mathrm{i}}^{\mathrm{M}} \mu_{\mathrm{i}}\right.$. In the exponential factor, $\overrightarrow{\mathrm{R}_{\mathrm{M}}}=\sum_{\mathrm{m}=1}^{\mathrm{M}} \overrightarrow{\mathrm{r}}_{\mu_{\mathrm{m}}}$. The bare states denoted by $\mid \Psi_{\mathrm{N}}^{(\mathrm{M})}(\overrightarrow{\mu)}>$ with $\mathrm{M}$ excitations can be expressed by means of the follows:

$$
\Psi_{\mathrm{N}}^{(\mathrm{M})}\left(\overrightarrow{\mu)}=\prod_{\mathrm{j}=1}^{\mathrm{M}}\left|\mathrm{e}_{\mu_{\mathrm{j}}}>\right| \mathrm{g}>^{\otimes(\mathrm{N}-\mathrm{M})} .\right.
$$

$\vec{\mu}$ is a vector with $\mathrm{M}$ components. Since a diagonalization the ensemble Hamiltonian including the cooperative interaction will introduce linear combination of these states expressed above, there are many alternatives to the representation for the ensemble basis states with $\mathrm{M}$ excited atonic states.

\section{Reduced-Density-Operator Approach}

A density-operator approach can provide an advantageous starting point for a non-perturbative and non-equilibrium quantum-statistical description of electromagnetic transitions. The quantum-open-systems (reduced-density-operator) approach $[4,15,16,28,29]$ can serve as a general framework for a fundamental microscopic description of the decoherence and relaxation processes, which arise from the influence of a much larger system. The much larger system is referred to as the environment. In the conventional reservoir approximation, the environment is assumed to be essentially unaffected by its interactions with the relevant quantum system of interest. Accordingly, in the reservoir approximation, the environment can be represented by a time-independent density operator.

A. Reduced-Density-Operator Description

Within the framework of the reduced-density-operator approach, the influence of the environment on the relevant quantum system of interest is treated stochastically, in terms of decoherence and relaxation processes together with spectral-line broadening mechanisms. These stochastic kinetics and spectral phenomena can be systematically and self-consistently investigated in terms of the Liouville-space self-energy corrections that are introduced in the complimentary time-domain (equation-of-motion) and frequency-domain (resolvent-operator) formulations of our reduced-density-operator approach [4].

A statistical state of the combined, interacting (closed) quantum system is conventionally assumed to be initially expressible as the uncorrelated, tensor-product of the separate density operators representing the relevant quantum system of interest and the environment. The quantum-statistical state of the relevant system, at an arbitrary time $t$, can be represented by means of the reduced, relevant density operator defined by $\rho^{r}(t)=\operatorname{Tr}_{E}\{\rho(t)\}$, where the quantumstatistical average (partial-trace operation) indicated by $\operatorname{Tr}_{\mathrm{E}}$ is to be taken over the large set of quantum numbers corresponding to the environmental degrees of freedom.

The partition of the entire, interacting quantum system into a relevant quantum system and an environment is inherently arbitrary and by no means apparent. In the ordinary Hilbertspace description, different divisions of the total Hamiltonian operator into a zero-order (unperturbed) Hamiltonian operator and an interaction (or perturbation) operator would be equivalent if the interaction could be incorporated to all orders. In contrast, different partitions in the reduced-densityoperator description are fundamentally inequivalent and will inevitably lead to dissimilar predictions. In this investigation, we point out the consequences of different partitions for radiative transitions of an atomic ensemble.

The reduced-density-operator description can be presented in compact forms by adopting the Liouville-space operator representation [30-36]. The Liouville-space operators are defined within a generalized Hilbert space, in which ordinary Hilbert-space operators, such as density operators, play the role of state vectors. The complete set of elementary Hilbertspace (density) operators of the form $|\alpha\rangle\langle\beta|$ provides a complete Liouville-space basis set. The elements of the complete Liouville-space basis set may be denoted, in terms of the two Hilbert-space state indices, using the Liouvillespace Dirac notation $|\alpha, \beta\rangle\rangle$. In this basis, the Liouville- 
space operators (which will be denoted by overbars in the following analyses) are represented by tetradic matrices, which are specified by four ordinary Hilbert-space state indices. The complex inner product $\left\langle\left\langle\rho_{1} \mid \rho_{2}\right\rangle\right\rangle$ of two Liouville-space state vectors $\left|\rho_{1}\right\rangle$ and $\left|\rho_{2}\right\rangle$ is defined as the trace operation $\operatorname{Tr}\left(\rho_{1}^{+} \rho_{2}\right)$, where the superscript “+” indicates the adjoint.

B. Reduced-Density-Operator Description in the Schrödinger picture

In the Schrödinger picture, the reduced density operator is treated as a time-dependent operator, and either a frequencydomain (resolvent-operator) formulation or a time-domain (equation-of-motion) formulation can be employed.
However, the operators corresponding to physical observables, such as those representing the quantized electromagnetic field, are treated as time-independent. In our semi-classical description of the electromagnetic response [4], the interaction or perturbation operator $\overline{\mathrm{V}}^{\mathrm{r}}$ corresponds to the classical electromagnetic field and is treated as a timedependent quantity.

1. Frequency-Domain (Resolvent-Operator) Formulation

In the frequency-domain formulation of our reduceddensity-operator description, the electromagnetic-transition rate for a general multi-photon transition can be expressed, in terms of the reduced, relevant Liouville-space transition operator $\overline{\mathrm{T}}^{\mathrm{r}}(+\mathrm{i} \varepsilon)$, in the generalized Fermi Golden-Rule form $[4,36,37]$ :

$$
A_{R}(i \rightarrow f)=-i \lim _{\varepsilon \rightarrow 0}\left\langle\left\langle P_{f}^{r}\left|\bar{T}^{r}(+i \varepsilon)\right| \rho_{i}^{r}\right\rangle\right\rangle=-i \lim _{\varepsilon \rightarrow 0}\left\langle\left\langle P_{f}^{r}\left|\bar{V}^{r}+\bar{V}^{r} \bar{G}^{r}(+i \varepsilon) \bar{V}^{r}\right| \rho_{i}^{r}\right\rangle\right\rangle .
$$

The reduced, relevant Liouville-space resolvent (or Green) operator is expressed by $\overline{\mathrm{G}}^{\mathrm{r}}(+\mathrm{i} \varepsilon)=\left[+\mathrm{i} \varepsilon-\overline{\mathrm{L}}^{\mathrm{r}}-\bar{\Sigma}(+\mathrm{i} \varepsilon)\right]^{-1}$, where $\overline{\mathrm{L}}^{\mathrm{r}}$ is the relevant Liouvillian operator and $\bar{\Sigma}(+\mathrm{i} \varepsilon)$ is the Liouvillespace self-energy operator. The tetradic-matrix elements
$\overline{\mathrm{L}}_{\alpha \beta \gamma \delta}^{\mathrm{r}}$ of the relevant Liouvillian operator $\overline{\mathrm{L}}^{\mathrm{r}}$ may be obtained by means of the following commutation relationships:

$$
\left\langle\alpha \overline{\mathrm{L}}^{\mathrm{r}} \rho^{\mathrm{r} \mid} \mid \beta\right\rangle=\left\langle\alpha\left|(1 / \hbar)\left[\mathrm{H}^{\mathrm{r}}, \rho^{\mathrm{r}}\right]\right| \beta\right\rangle=\sum_{\gamma} \sum_{\delta} \overline{\mathrm{L}}_{\alpha \beta, \gamma}^{\mathrm{r}} \rho_{\gamma \delta}^{\mathrm{r}}=\sum_{\gamma} \sum_{\delta}(1 / \hbar)\left(\mathrm{H}_{\alpha \gamma}^{\mathrm{r}} \delta_{\delta \beta}-\delta_{\alpha \gamma} \mathrm{H}_{\delta \beta}^{\mathrm{r}}\right) \rho_{\gamma \delta}^{\mathrm{r}} .
$$

The relevant Hamiltonian operator $\mathrm{H}^{\mathrm{r}}$ describes the many-particle quantum system together with the quantized electromagnetic field. The relevant Liouvillian operator $\overline{\mathrm{L}}^{\mathrm{r}}$ can be decomposed as $\overline{\mathrm{L}}^{\mathrm{r}}=\overline{\mathrm{L}}_{0}^{\mathrm{r}}+\overline{\mathrm{V}}^{\mathrm{r}}$, where $\overline{\mathrm{L}}_{0}{ }^{\mathrm{r}}$ is the zeroorder (unperturbed) relevant Liouvillian operator corresponding to the zero-order relevant Hamiltonian operator $\mathrm{H}_{0}{ }^{\mathrm{r}}$ and the Liouville-space perturbation operator $\overline{\mathrm{V}}^{\mathrm{r}}$ describes the relevant electromagnetic interaction. Eq.
(23) provides a more general definition of photon density matrix $\rho^{\mathrm{R}}$ than is possible using only the polarization density matrix introduced by Eq. (1).

The reduced, relevant Liouville-space transition operator can be more conveniently evaluated, in terms of the zeroorder reduced, relevant Liouville-space resolvent (or Green) operator $\overline{\mathrm{G}}_{0}{ }^{\mathrm{r}}(+\mathrm{i} \varepsilon)=\left[+\mathrm{i} \varepsilon-\overline{\mathrm{L}}_{0}{ }^{\mathrm{r}}-\bar{\Sigma}(+\mathrm{i} \varepsilon)\right]^{-1}, \quad$ as $\quad$ a perturbation expansion in powers of $\overline{\mathrm{V}}^{\mathrm{r}}$ :

$$
\overline{\mathrm{T}}^{\mathrm{r}}(+\mathrm{i} \varepsilon)=\overline{\mathrm{V}}^{\mathrm{r}}+\overline{\mathrm{V}}^{\mathrm{r}} \overline{\mathrm{G}}_{0}^{\mathrm{r}}(+\mathrm{i} \varepsilon) \overline{\mathrm{V}}^{\mathrm{r}}+\overline{\mathrm{V}}^{\mathrm{r}} \overline{\mathrm{G}}_{0}^{\mathrm{r}}(+\mathrm{i} \varepsilon) \overline{\mathrm{V}}^{\mathrm{r}} \overline{\mathrm{G}}_{0}^{\mathrm{r}}(+\mathrm{i} \varepsilon) \overline{\mathrm{V}}^{\mathrm{r}}+\overline{\mathrm{V}}^{\mathrm{r}} \overline{\mathrm{G}}_{0}^{\mathrm{r}}(+\mathrm{i} \varepsilon) \overline{\mathrm{V}}^{\mathrm{r}} \overline{\mathrm{G}}_{0}^{\mathrm{r}}(+\mathrm{i} \varepsilon) \overline{\mathrm{V}}^{\mathrm{r}} \overline{\mathrm{G}}_{0}^{\mathrm{r}}(+\mathrm{i} \varepsilon) \overline{\mathrm{V}}^{\mathrm{r}} \ldots
$$

As a first approximation, we may retain only the lowestorder non-vanishing contribution to the electromagnetictransition rate for a specific n-photon process of interest. Higher-order contributions will give rise to QED radiative corrections. For a single-photon transition, the lowest-order contribution will be given by $\overline{\mathrm{V}}^{\mathrm{r}} \overline{\mathrm{G}}_{0}{ }^{\mathrm{r}}(+\mathrm{i} \varepsilon) \overline{\mathrm{V}}^{\mathrm{r}}$. In the case of a two-photon transition, the lowest-order contribution will be provided

by $\overline{\mathrm{V}}^{\mathrm{r}} \overline{\mathrm{G}}_{0}{ }^{\mathrm{r}}(+\mathrm{i} \varepsilon) \overline{\mathrm{V}}^{\mathrm{r}} \overline{\mathrm{G}}_{0}{ }^{\mathrm{r}}(+\mathrm{i} \varepsilon) \overline{\mathrm{V}}^{\mathrm{r}} \overline{\mathrm{G}}_{0}{ }^{\mathrm{r}}(+\mathrm{i} \varepsilon) \overline{\mathrm{V}}^{\mathrm{r}}$. In the spectral description of light-matter interactions, it may be necessary to take into account an extensive class of single-photon and multi-photon transitions.

a. Frequency-Domain Liouville-Space Self-Energy Operator
The frequency-domain Liouville-space self-energy operator $\bar{\Sigma}(+\mathrm{i} \varepsilon)$ can be expressed in terms of the Zwanzig Liouville-space projection operators $\left.\overline{\mathrm{P}}=\left|\rho^{\mathrm{E}}\right\rangle\right\rangle\left\langle\left\langle\mathrm{I}^{\mathrm{E}}\right|\right.$ and $\overline{\mathrm{Q}}=1-\overline{\mathrm{P}}$, where $\mathrm{I}^{\mathrm{E}}$ denotes the environmental identity operator $[30,31]$. The projection operator $\overline{\mathrm{P}}$ projects onto the subspace of states for the relevant-system degrees of freedom (uncorrelated with the environmental degrees of freedom), while complementary projection operator $\bar{Q}$ projects onto the orthogonal subspace of states for the irrelevant (environmental) degrees of freedom (taking into account the system-environment correlations). The frequency-domain Liouville-space self-energy operator $\bar{\Sigma}(\mathrm{z})$ can then be expressed in the forms $[4,36,37]$ :

$$
\bar{\Sigma}(\mathrm{z})=\overline{\mathrm{P}} \overline{\mathrm{V}}^{\mathrm{ir}} \overline{\mathrm{P}}+\overline{\mathrm{P}} \overline{\mathrm{V}} \overline{\mathrm{Q}} \frac{1}{\mathrm{z}-\overline{\mathrm{Q}} \overline{\mathrm{L}} \overline{\mathrm{Q}}} \overline{\mathrm{Q}} \overline{\mathrm{V}} \overline{\mathrm{P}}=\operatorname{Tr}_{\mathrm{E}}\left\lfloor\left(\overline{\mathrm{V}}^{\mathrm{ir}}+\overline{\mathrm{V}} \overline{\mathrm{Q}} \frac{1}{\mathrm{z}-\overline{\mathrm{Q}} \overline{\mathrm{L}} \overline{\mathrm{Q}}} \overline{\mathrm{Q}} \overline{\mathrm{V}}\right) \rho^{\mathrm{E}}\right\rfloor .
$$


The complete Liouvillian operator $\overline{\mathrm{L}}$ is defined in terms of the total Hamiltonian operator for the entire (closed) interacting quantum system. The total Liouville-space interaction operator $\bar{V}$ is partitioned as $\bar{V}=\bar{V}^{r}+\bar{V}^{\text {ir }}$, where the irrelevant Liouville-space interaction operator $\bar{V}^{\text {ir }}$ includes the environmental interactions. A major advantage of the Liouville-space operator representation is that a fundamental microscopic treatment can be provided for the environmental interactions, on an equal footing with the QED radiative corrections, which arise from virtual transitions involving the creation and annihilation of electron-positron pairs together with photons. In many descriptions, the environmental interactions are represented by phenomenological parameters.

$$
\left.\left.\left|\rho_{i}^{r}\right\rangle\right\rangle=\sum_{a} \sum_{a^{\prime}} \sum_{\left\{n_{i}\right\}} \sum_{\left.n_{i}^{\prime}\right\}}\left|a^{\prime},\left\{n_{i}\right\}\left\{n_{i}^{\prime}\right\}\right\rangle\right\rangle\left\langle\left\langle\mathrm{aa}^{\prime} \mid \rho_{i}^{s}\right\rangle\right\rangle\left\langle\left\langle\left\{n_{i}\right\}\left\{n_{i}^{\prime}\right\} \mid \rho_{i}^{R}\right\rangle\right\rangle,
$$

$$
\left.\left.\left|\mathrm{P}_{\mathrm{f}}^{\mathrm{r}}\right\rangle\right\rangle=\sum_{\mathrm{b}} \sum_{\left\{\mathrm{n}_{\mathrm{i}}^{\prime}\right\}}\left|\mathrm{bb},\left\{\mathrm{n}_{\mathrm{i}}^{\prime}\right\}\left\{\mathrm{n}_{\mathrm{i}}^{\prime}\right\}\right\rangle\right\rangle
$$

Note that the indices that are used as subscripts on the reduced density operator and on the projection operator are intended to denote, respectively, the initial and final states of the relevant many-particle and many-photon quantum system in the electromagnetic transition, while the index $\mathrm{i}$ that is used as a subscript on the photon occupation numbers $n$ and $\mathrm{n}^{\prime}$ will be understood to denote the various modes of the quantized electromagnetic field. Note that the various photon modes are specified by the propagation vector and the polarization. We emphasize that the general reduced-densityoperator description is applicable to non-equilibrium quantum-statistical distributions for the charged particles and the electromagnetic fields, which can be obtained starting from the time-domain (equation-of-motion) formulation of our reduced-density-operator description presented below. The general expression for the final-state projection operator given by Eq. (28) can be adapted to define the photonpolarization density operator that will replace the simplified expression given by Eq. (1).

Eq. (27) has been simplified by introducing the conventional assumption that the initial-state reduced density operator can be expressed as a tensor product of the separate initial-state density operators for the isolated charged-particle system (S) and for the relevant radiation field (R), i. e., $\rho_{i}^{r}=\rho_{i}^{S} \otimes \rho_{i}^{R}$. Note that, in this eigenstate decomposition, both the initial matter-state coherences (corresponding to the non-diagonal reduced-density-matrix elements with $a \neq \mathrm{a}^{\prime}$ ) and the analogous initial-state electromagnetic-field correlations (involving non-diagonal reduced-density-matrix elements with $\left\{n_{i}\right\} \neq\left\{n_{i}^{\prime}\right\}$ ) have been taken into account.

When the system-environment interactions are sufficiently weak, the Liouville-space self-energy operator $\bar{\Sigma}$ may be b. Initial and Final States in the Electromagnetic Transitions

In our description of an extensive class of single-photon (n $=1)$ and multi-photon $(n>1)$ processes, the initial-state reduced density operator $\rho_{i}{ }^{r}$ can be represented in terms of the tensor-product eigenstates $|\alpha\rangle=\left|\mathrm{a},\left\{\mathrm{n}_{\mathrm{i}}\right\}\right\rangle=|\mathrm{a}\rangle \otimes\left|\left\{\mathrm{n}_{\mathrm{i}}\right\}\right\rangle$. The relevant final-state projection operator $\mathrm{P}_{\mathrm{f}}{ }^{\mathrm{r}}$ projects onto the subspace of tensor-product eigenstates formed from the unperturbed many-electron eigenstates $|b\rangle$ that can be created as a result of an electromagnetic transition. Accordingly, the required operator eigenstate decompositions can be expressed as follows:

expanded in a perturbation series involving increasing powers of the total Liouville-space interaction operator $\overline{\mathrm{V}}$. Retaining only the lowest-order non-vanishing contribution, which corresponds to the Born approximation, the total spectral-line shift and width in the diagonal-resolvent approximation can be reduced to the sums of the partial contributions from elementary collisional and radiative processes acting alone [4]. Interference between transition amplitudes can occur in the high-order contributions to the width and shift, as well as in our general tetradic-matrix expression, which is valid for overlapping spectral lines.

2. Time-domain (Equation-of-Motion) Formulation

The time-domain (equation-of-motion) formulation of the reduced-density-operator description is based on the generalized Master equation [36, 37]:

$$
\frac{\partial}{\partial t} \rho^{r}(t)=-i \bar{L}^{r}(t) \rho^{r}(t)-i \int_{t_{0}}^{t} d t^{\prime} \bar{\Sigma}\left(t, t^{\prime}\right) \rho^{r}\left(t^{\prime}\right)
$$

This closed-form equation of motion for the reduced, relevant density operator $\rho^{r}(t)=\bar{P} \rho(t)$ has been derived by neglecting the initial-state correlations. Initial-state correlations are automatically excluded by the conventional assumption that the entire initial-state density operator for the combined, interacting light-matter system can be represented as an uncorrelated, tensor product of individual density operators for the separate, isolated subsystems. The photon density matrix obtained by means of Eq. (29) can provide a more general time-dependent description than the photon density matrix $\rho^{\mathrm{R}}(\mathrm{t})$ that is obtained from Eq. (16).

a. Time-Domain Self-Energy Operator

The time-domain Liouville-space self-energy operator kernel $\bar{\Sigma}\left(\mathrm{t}, \mathrm{t}^{\prime}\right)$ can be formally expressed by means of the relationships [36, 37]:

$$
\bar{\Sigma}\left(\mathrm{t}, \mathrm{t}^{\prime}\right)=-\mathrm{i} \overline{\mathrm{P}} \overline{\mathrm{V}}(\mathrm{t}) \overline{\mathrm{Q}} \overline{\mathrm{g}}_{\mathrm{Q}}\left(\mathrm{t}, \mathrm{t}^{\prime}\right) \overline{\mathrm{Q}} \overline{\mathrm{V}}\left(\mathrm{t}^{\prime}\right) \overline{\mathrm{P}}=-\mathrm{i} \operatorname{Tr}_{\mathrm{E}}\left[\overline{\mathrm{V}}(\mathrm{t}) \overline{\mathrm{Q}}_{\mathrm{Q}}\left(\mathrm{t}, \mathrm{t}^{\prime}\right) \overline{\mathrm{Q}} \overline{\mathrm{V}}\left(\mathrm{t}^{\prime}\right) \mathrm{p}^{\mathrm{E}}\right]
$$


The Q-subspace projection $\overline{\mathrm{g}}_{\mathrm{Q}}\left(\mathrm{t}, \mathrm{t}^{\prime}\right)$ of the Liouvillespace propagator is defined, in terms of the time-ordering operator $T$, as follows:

$$
\overline{\mathrm{g}}_{\mathrm{Q}}\left(\mathrm{t}, \mathrm{t}^{\prime}\right)=T \exp \left[-\mathrm{i} \int_{\mathrm{t}^{\prime}}^{\mathrm{t}} \mathrm{dt^{ \prime \prime }} \overline{\mathrm{Q}} \overline{\mathrm{L}}\left(\mathrm{t}^{\prime \prime}\right) \overline{\mathrm{Q}}\right] .
$$

In contrast to the propagator $\bar{g}\left(\mathrm{t}, \mathrm{t}^{\prime}\right)$ corresponding to the total Liouvillian operator $\overline{\mathrm{L}}$ for the closed interacting lightmatter system, which describes unitary (reversible) time evolution, the Q-subspace projection describes non-unitary (irreversible) time evolution. The Liouville-space self-energy operator kernel $\bar{\Sigma}\left(\mathrm{t}, \mathrm{t}^{\prime}\right)$, which appears in the time-domain (equation-of-motion) formulation, can be related to the timeindependent Liouville-space self-energy operator $\bar{\Sigma}(\mathrm{z})$, occurring in the frequency-domain (resolvent-operator) formulation. This relationship may be viewed in terms of the connection between the time-domain propagator and corresponding frequency-domain resolvent operator, which can be most generally expressed in the contour-integration form:

$$
\overline{\mathrm{g}}\left(\mathrm{t}, \mathrm{t}^{\prime}\right)=\lim _{\varepsilon \rightarrow 0} \frac{1}{2 \pi \mathrm{i}} \oint \mathrm{dzexp}\left[-\mathrm{iz}\left(\mathrm{t}-\mathrm{t}^{\prime}\right)\right] \overline{\mathrm{G}}(\mathrm{z}+\mathrm{i} \varepsilon),
$$

where the general contour integration can be evaluated as a Fourier or a Laplace transformation.

In the commonly adopted Markov (short-memory-time) approximation, the Liouville-space self-energy operator kernel $\bar{\Sigma}\left(\mathrm{t}, \mathrm{t}^{\prime}\right)$ is assumed to be independent of time. The Markov approximation can be introduced into the equation of motion for the reduced, relevant density operator $\rho^{r}(t)$ by utilizing the relationship:

$$
\bar{\Sigma}\left(\mathrm{t}, \mathrm{t}^{\prime}\right)=\lim _{\mathrm{z} \rightarrow \mathrm{i} 0} \bar{\Sigma}(\mathrm{z}) \delta\left(\mathrm{t}-\mathrm{t}^{\prime}\right) .
$$

In this approximation, the corresponding frequencydomain Liouville-space self-energy operator $\bar{\Sigma}(\mathrm{i} 0)$, which will be denoted simply by $\bar{\Sigma}$, is independent of the frequency. For a completely consistent treatment of the nonMarkovian dynamics, it may be necessary to retain the initial-state correlation term that was excluded in the derivation of the generalized Master equation.

b. Many-Particle-System and Electromagnetic-Field Equations

An equation of motion for the many-particle-system density operator can be derived from Eq. (29) by performing the additional average (partial-trace) operation over the photon states. On the other hand, the dynamical equation for the density operator representing the quantum-statistical state of the electromagnetic field can be obtained from Eq. (29) by carrying out the complimentary additional average (partialtrace) operation over the many-particle-system states.

Quantum-mechanical interference terms will be encountered in the evaluation of the tetradic matrix elements of the Liouville-space self-energy operator kernel that appears in the equation of motion for the many-particlesystem density operator. After introducing the Born (lowestorder) and Markov (short-memory-time) approximations, the set of dynamical equations for the many-particle-system state-population densities (corresponding to the diagonal density-matrix elements) can be expressed in terms of the familiar (lowest-order) radiative and non-radiative transition rates that are obtained from an evaluation of the standard Fermi Golden-Rule formula of ordinary Hilbert-space perturbation theory. The optical Bloch equations are usually understood to correspond to the extended set of densitymatrix equations, taking into account the many-particlesystem state coherences (corresponding to the non-diagonal density-matrix elements).

C. Reduced-Density-Operator Description in the Heisenberg Picture

In the Heisenberg picture, the reduced density operator is treated as a time-independent operator, while operators corresponding to observable quantities, such as those representing the quantized electromagnetic field, are treated as time-dependent. It is convenient to introduce a set of timedependent operators $\left\{\mathrm{G}_{\mu}(\mathrm{t})\right\}$ corresponding to observables on a quantum system [28, 38]. For examples, the macroscopic electromagnetic fields can be obtained by choosing the time-dependent operators to be the microscopic electromagnetic-field operators. It is well known that the dynamical equations for the macroscopic electromagnetic fields that are derived from the quantized-field operators have the same form as the Maxwell Equations of classical electromagnetic theory, provided that the microscopic charge density and current density operators (occurring in the equations for the microscopic electromagnetic-field operators) are allowed to have their most general non-local and non-linear forms. It is also well known that the corresponding definition in the Schrödinger picture should yield the same expectation values, when the density operator pertains to the entire interacting (closed) light-matter system. The analysis may be more advantageous in one of the two alternative pictures. For an open quantum system described by a reduced density operator, however, different expectations values may be obtained in the two alternative pictures. If this is the case, then the choice of the picture (that is adopted for the description of the non-unitary time evolution) will have a significant effect on the predictions that are obtained for various physical properties and phenomena.

D. Hierarchical Reduced-Density-Operator Formulations for Many-Body Systems

In the treatment of many-electron quantum correlations within the framework of the reduced-density-operator approach, it may be necessary to introduce a correlation (or cluster) decomposition for the reduced density operator, together with a set (hierarchy) of coupled equations of motion for the various correlation components. For example, the equation of motion for the single-electron reduced density operator would be coupled to that for the two-electron reduced density operator. This coupling is obviously a consequence of 
the electron-electron interaction. The introduction of this set (hierarchy) of coupled reduced-density-operator equations of motion is expected to be essential for the practical description of solid-state systems, for which the number of electrons is very large in comparison with that for atomic systems. In order to treat a quantized electromagnetic field on an equal footing with the quantized electronic system, it will be necessary to introduce a generalized correlation (or cluster) decomposition, together with a generalized correlation hierarchy of coupled reduced-density-operator equations of motion. In this generalization, quantum correlations involving the photons would be included, on an equal footing with the chargedparticle correlations. A further generalization would be necessary to take into account correlations involving phonons. In the reduced-density-matrix approach, it will be necessary to separate the many-body correlations that must be treated as a part of a fully correlated quantum system from those interactions (if any) that can be adequately treated as environmental effects.

\section{E. Perturbation-Theory Treatments}

In the Schrödinger picture, the reduced-density operator may be expanded in a perturbation-theory series, in increasing powers of the electromagnetic-interaction operator. An infinite set (hierarchy) of coupled integrodifferential equations can be obtained for the electromagnetic-field components of the reduce-densityoperator describing the matter system, and another (complimentary) infinite set (hierarchy) of coupled equations can be derived for the time-independent operators. In our semi-classical reduced-density-operator description of the electromagnetic interaction [4], the electromagnetic-field components are labeled by the powers of the electric-field components of the classical electromagnetic field. In the quantized-field description, a spatial photon-mode representation can be introduced, such as the plane-wave expansions. In the alternative Heisenberg picture, each of the time-dependent operators in the selected set of the physical observables may be expanded in a perturbation-theory series.

\section{Conclusions and Future Plans}

In Section II, we have presented a steady-state (timeindependent) polarization density-matrix description of superradiant emission, emphasizing that a realistic treatment of the superradiant emission process it expected to require the incorporation of initial-state coherences. The evaluation of the expression for the polarization density-matrix will also require a detailed knowledge of the cooperative interactions among the individual many-electron atomic systems comprising the ensemble. In Section III, a time-dependent description of superradiant emission has been presented. The time-dependent description is expected to be necessary for a more general characterization of the angular distribution and polarization of the superradiant emission under general excitation conditions. In Section IV, we discussed the representation of the atomic-ensemble states. In Section V, we presented a more comprehensive reduced-density- operator approach, considering the influence of environmental decoherence and relaxation processes on both the atomic-ensemble states and the spectral-lines shapes. Reduced-density-operator descriptions have been previously employed for the description of superradiant emission in various many-electron ensembles [40]. A closely related phenomenon, known as superabsorption, has also been described [40]. Finally, the suppression of spontaneous emission for coherent momentum transfer has been observed experimentally [43].

\section{Acknowledgements}

I would like to thank Wesley Campbell for suggesting this topic and for helpful discussions. Financial support for this work has been provided by The Office of Naval Research through the Basic Research Program at The Naval Research Laboratory.

\section{References}

[1] R. H. Dicke, Phys. Rev. 93, 99 (1954).

[2] W. Guerin, M. O. Araujp, and R. Kaiser, Phys. Rev. Lett. 116, 083601 (2016).

[3] V. L. Jacobs and A. B. Filuk, Phys. Rev. A 60, 1975 (1999).

[4] V. L. Jacobs, Phys. Rev. A 90, 033841 (2014).

[5] J. R. Oppenheimer, Z. Phys. 43, 27 (1927).

[6] I. C. Percival and M. J. Seaton, Phil. Trans. R. Soc. A 251, 113 (1958).

[7] M. K. Inal and J. Dubau, J. Phys. B 20, 4221 (1987).

[8] M. K. Inal and J. Dubau, J. Phys. B 22, 3329 (1989).

[9] A. S. Shlyaptseva, A. M. Urnov, and A. V. Vinogradov, P. N. Lebedev Physical Institute of the U. S. S. R. Academy of Sciences Report No. 194, 1981.

[10] A. V. Vinogradov, A. M. Urnov, and A. S. Shlyaptseva, in Atomic and Ionic Spectra and Elementary Processes in Plasmas, Proceedings of the P. N. Lebedev Physics Institute, Academy of Sciences of Russia, Vol. 912, Edited by I. I. Sobelman (Nova Science Publishers, Commack, New York, 1992), 93

[11] A. S. Shlyaptseva et al., Phys. Rev. A 57, 888 (1998).

[12] M. F. Gu, D. W. Savin, and P. Beiersddorfer, J. Phys. B 32, 5371 (1999).

[13] V. L. Jacobs, J. Phys. B 5, 2257 (1972).

[14] V. L. Jacobs, J. Phys. B 6, 1461 (1973).

[15] U. Fano, Rev. Mod. Phys. 29, 74 (1957).

[16] K. Blum, Density Matrix Theory and Applications, Second Edition, (Plenum, New York, 1996).

[17] S. A. Kazantsev and J.-C. Hénoux, Polarization Spectroscopy of Ionized Gases, (Kluwer, Dordrecht, 1995). 
[18] U. Fano and G. Racah, Irreducible Tensorial Sets, (Academic, New York, 1959).

[19] V. B. Berestetskii, E. M. Lifshitz, and L. P. Pitaevskii, Quantum Electrodynamics, (Pergamon, Oxford, 1982).

[20] W. Happer, Ann. Phys. 48, 579 (1968).

[21] W. Happer, Rev. Mod. Phys. 44, 169 (1972).

[22] A. Omont, Progress in Quantum Electronics 5, 69 (1977).

[23] W. E. Baylis, in Progress in Atomic Spectroscopy, edited by W. Hanle and H. Kleinpoppen (Plenum, New York, 1979).

[24] V. L. Jacobs, P. G. Burke, and R. M. Potvliege, J. Phys. B: At. Mol. Opt. Phys. 47, 025401 (2014).

[25] H. H. Jen, Phys Rev A, 96023814 (1917).

[26] E. Fick and G. Sauermann, The Quantum Statistics of Dynamic Processes (Springer, Berlin, 1990).

[27] C. Cohen-Tannoudji, J. Dupont-Roc, and G. Grynberg, AtomPhoton Interactions (Wiley-Interscience, New York, 1992).

[28] R. Zwanzig, in Lectures in Theoretical Physics III, edited by W. E. Brittin, D. W. Downs and J. Downs (Interscience, New York, 1961).
[29] R. Zwanzig, J. Chem. Phys. 33, 1338 (1960).

[30] U. Fano, Phys. Rev. 131, 259 (1963).

[31] U. Fano, in Lectures on Many-Body Problems, edited by E. R. Caianiello (Academic Press, New York, 1964).

[32] A. Ben-Reuven, Adv. Chem. Phys. 33, 235 (1975).

[33] S. Mukamel, Phys. Rep. 93, 1 (1982).

[34] V. L. Jacobs, J. Cooper, and S. L. Haan, Phys. Rev. A 50, 3005 (1994).

[35] V. L. Jacobs, Canadian Journal of Physics 89, 533 (2011).

[36] A. Ben-Reuven and Y. Rabin, Phys. Rev. A 19, 2056 (1979).

[37] K. Burnett, J. Cooper, R. J. Ballagh, and E. W. Smith, Phys. Rev. A 22, 2005 (1980).

[38] H. Mori, Prog. Theor. Phys. 33, 423 (1965).

[39] M. Gross and S. Haroche, Physics Reports 93, 5 (1982).

[40] K. D. P. Higgins et al, Nature Communications 5, 4705 (2014).

[41] Xueping Long et al, Phys. Rev, Letters 123, 033603 (2019). 